\title{
Factors Related to the Choice of Family Medicine: A Reassessment and Literature Review
}

\author{
Janet H. Senf, PhD, Doug Campos-Outcalt, MD, MPA, and Randa Kutob, MD
}

Background: Recent decreases in the number of students entering family medicine has prompted reconsideration of what is known about the factors affecting specialty choice.

Metbods: Thirty-six articles on family medicine specialty choice published since 1993 were reviewed and rated for quality.

Results: Rural background related positively and parents' socioeconomic status relates negatively to choice of family medicine. Career intentions at entry to medical school predict specialty choice. Students who believe primary care is important, have low income expectations, and do not plan a research career are more likely to choose family medicine. The school characteristic related to choice of family medicine is public ownership. Large programs to increase numbers entering primary care seem effective. Required family medicine time in clinical years is related to higher numbers selecting family medicine. Faculty role models serve both as positive and negative influences. Students rejecting family medicine are concerned about prestige, low income, and breadth of knowledge required. Students planning on a career in a disadvantaged or rural area are more likely to enter family medicine.

Conclusions: Multiple factors are consistently shown to be related to the choice of the specialty of family medicine. (J Am Board Fam Pract 2003;16:502-12.)

More than 2 decades ago, the Graduate Medical Education Advisory Committee issued a report predicting an excess of specialists and a need to produce more generalist physicians. ${ }^{1}$ In the years since, considerable attention has been focused on how to address the continuing need for more generalist physicians, both to provide health care for the more than $20 \%$ of the nation who live in rural $\operatorname{areas}^{2}$ and to fill the continuing demand for physicians who can function as the entry point to the medical care system.

Much of this ongoing scrutiny is directed at the nation's medical schools to determine what can be done to increase their output of generalist physicians. Efforts to influence medical schools have included state legislation requiring or suggesting a certain level of primary care output, ${ }^{3}$ the develop-

Submitted, revised, 5 Feb 2003.

From the University of Arizona Department of Family and Community Medicine, Tucson, Arizona (JHS, RK), and Maricopa County Department of Public Health and University of Arizona Department of Family and Community Medicine, Phoenix, Arizona (DCO). Address correspondence to Janet H. Senf, PhD, Department of Family and Community Medicine, 1450 N. Cherry Ave., Tucson, AZ 85719 (e-mail: jsenf@u.arizona.edu).

Funding for this project came from the American Academy of Family Physicians contract 420350, "Factors Influencing Medical Students in their Choice of Family Medicine as a Specialty." ment of special task forces that have generated recommendations on ways to increase the output of primary care physicians, ${ }^{4}$ and significant funding from the federal government and private foundations to develop curricula or new training modalities intended to increase the number of graduating generalist physicians. $^{5-7}$ During the years from 1993 to 1997, it seemed that these efforts were having an effect; the proportion of US medical school graduates selecting family medicine increased in each year, reaching $17.3 \%$ in 1997. However, beginning in 1998, there has been a decline in each year, with just $10.5 \%$ selecting family medicine in $2002 .^{8}$

As a result of the interest in ways to increase the numbers of physicians in primary care, a substantial body of research on specialty choice has developed. There have been 3 reviews since 1995. Bland et $\mathrm{al}^{9}$ and Meurer ${ }^{10}$ covered most factors related to specialty choice. The review by Campos-Outcalt et $\mathrm{al}^{11}$ was limited to just 3 areas, curricula, role models and school funding. ${ }^{10}$ The conclusions of these 3 reviews, which included research published through 1993, serve as the starting point for this one, which critically reviews the literature published since 1993 on the choice of family medicine as a specialty. Table 1 presents the conclusions from these reviews as well as the findings from this 
review. A fourth, self-described, review by Reed ${ }^{12}$ used an illustrative selection of studies and could therefore not draw conclusions about the comprehensive body of research. In addition, there was one comprehensive look at factors that influence specialty choice. However, the method for selection of the literature included was not discussed, so it is not known to what extent the literature used was allinclusive or was selected to support key points of discussion. ${ }^{13}$

\section{Methods}

A literature search was conducted using MEDLINE, PsychINFO, HealthSTAR, Expanded Academic Index, and the Health and Psychosocial Instruments. Keywords and their intersections included family practice, family medicine, career choice, specialty choice, primary care, and medical student. In addition, the bibliographies from the articles obtained were compared with the list to locate additional articles.

Articles were reviewed by the authors to determine whether they included original research related to choice of family medicine as a specialty. The final set of articles were read and scored by at least 2 of the authors, using a ranking system that has been described in detail previously. ${ }^{10}$ This system of scoring assigns up to 10 points each for: 1) type of study design, 2) size of the study, 3) response rate, 4) number of years studied, 5) data sources, 6) statistical analysis, and 7) theoretical basis of the research. The total number of points possible is 70. Differences in scoring were resolved either by a review by a third author or by discussion among the authors. More than 150 articles were reviewed; 36 articles met the inclusion criteria and were scored.

In this review, the content of the articles has been arranged in a roughly chronological fashion, similar to the natural history of specialty choice described by Rosenthal. ${ }^{14}$ Data on the characteristics that medical students have on entrance to medical school are presented first, including their personal characteristics and background, their values and their intentions. This first set is often of interest to educators who would like to be able to select applicants who will ultimately go into one of the primary care specialties. Information on medical school characteristics is reviewed next; generally, these are the relatively fixed elements of the school (eg, the ownership of the school). The third group of articles represents data on experiences that occur during the process of medical education, including the curriculum and informal culture of the school, values that may be expressed during this process, and information on decision-making about specialty. The final group represents what can be seen as the outcomes of the process of medical education, including perceptions about the specialties, career plans, and the influence of debt.

\section{Results: What Do We Know and What Have We Learned Since 1993?}

The mean quality score for the 36 articles reviewed was 33.2, which was somewhat higher than the mean of 29.9 found in $1995 .^{10}$ The range of scores was from 14 to 48 , which includes from $20 \%$ to $69 \%$ of the available points. Articles that scored below 20 (less than $25 \%$ of the available points) were not included in this review; when findings are contradictory, the quality of the study determined which results should receive more weight. Overall quality scores were not significantly related to year of publication; ie, the overall quality of research in this area has not improved progressively during the more recent time period but overall is better than the first half of the decade. One important change, however, is the larger number of studies using multivariate analyses, with the negative results presented as well as the positive findings. Multivariate analysis helps to differentiate between those factors that are directly related to the choice of a particular specialty and factors that are related only indirectly through an intervening variable. Presenting negative results is especially important, because generally negative findings are under-represented in the published literature, leading to an overestimation of the importance of a given factor.

\section{Student Characteristics}

Gender

Four studies found higher proportions of women than men selecting family medicine but none of the differences were statistically significant. ${ }^{14-17}$ Three additional studies either found equal numbers or fewer women entering family medicine. ${ }^{18-20}$ Three studies reported multivariate analyses with at least one other control variable. Of these, 2 did not find that gender predicted choice of family medicine, ${ }^{21,22}$ whereas the other found that being female 


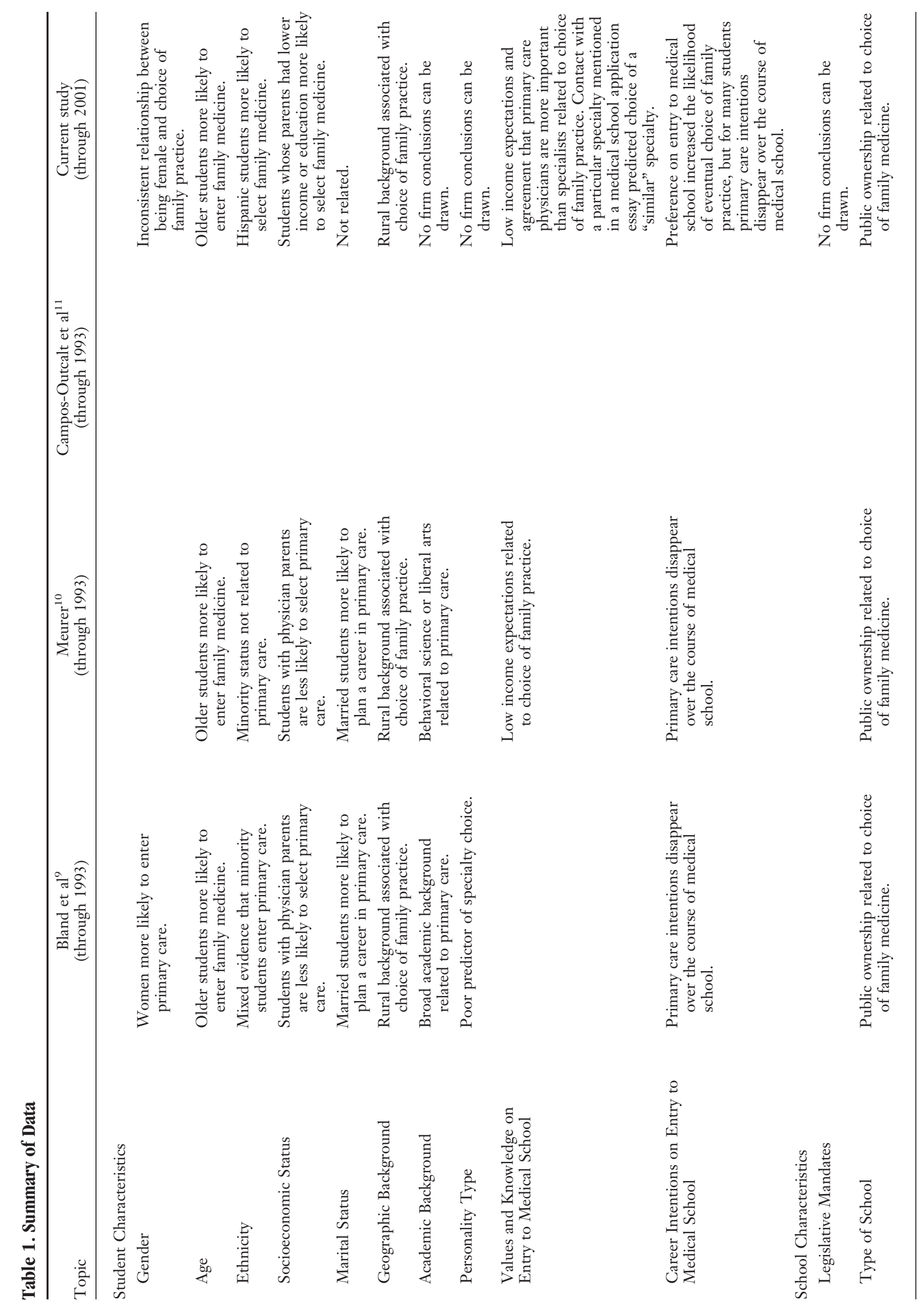



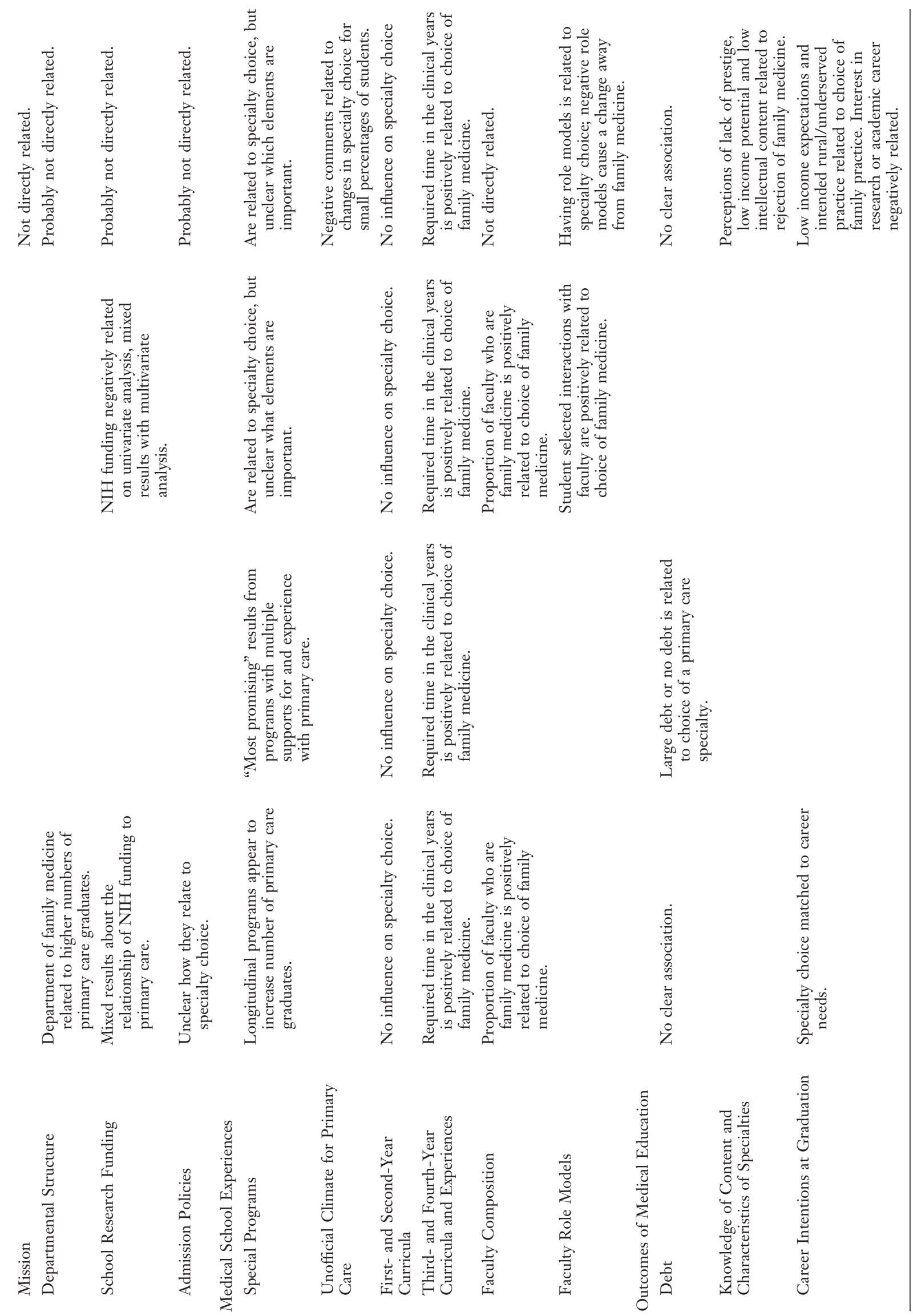
modestly increased the odds of choosing family practice. ${ }^{23}$ All 3 of these studies had relatively high quality scores, so that the results of one are not clearly more sound than the others.

Based on all the studies since the last review, it would seem that at this point, female gender is very slightly associated with but not consistently predictive of a career in family practice. Because the positive and negative findings were scattered over the years since 1993, it does not seem that there is a trend over time in the proportion of women entering family medicine.

\section{Age}

Two studies looking at age had conflicting results; one found that older age was associated with an interest in family medicine ${ }^{24}$ and the other found no association. ${ }^{25}$ Studies using multivariate analysis also had conflicting results; one did not find that age predicted plans for a family medicine career, ${ }^{22}$ the other found a linear positive relationship between age and a plan for a family medicine career. ${ }^{23}$ Although these results are mixed, it would seem that older age is at least weakly related to a choice of family medicine.

\section{Ethnicity}

Two studies since the previous reviews have examined ethnicity. ${ }^{22,26}$ The data from these studies provide limited support for the belief that Hispanics are more likely to select family medicine.

\section{Socioeconomic Status}

Two studies examined having physician parents; one with multivariate analysis found it was not a significant predictor of plans for family medicine, ${ }^{22}$ and the other with univariate analysis found it was. ${ }^{24}$ One study found that father's lower socioeconomic status was a predictor of family medicine, ${ }^{22}$ and another reported that parents' income over $\$ 100,000$ decreased the likelihood of plans for a family practice specialty, but parents' education was not significantly related. ${ }^{23}$

Although each of these studies used different measures of socioeconomic status, for all studies, the direction of influence was the same, albeit not particularly strong. The most consistent finding is that lower socioeconomic status or lower parental income or education is related to a choice of family medicine.

\section{Marital Status}

In the 2 studies reporting on marital status since 1993, one found a higher proportion of never married among those never interested in family medicine compared with those maintaining an interest (significance levels not given), ${ }^{24}$ and the other found that marital status (coded married/not married) did not predict plans for any of the 3 primary care specialties. ${ }^{22}$ Both of these studies had relatively high quality scores; however, the latter was multivariate, including controls for age, lending credibility to the idea that marital status is related to some other variable that in turn is the better predictor of choice of family medicine.

\section{Geographic Background}

All 3 studies that have been done since 1993 found that rural background is related to choice of family medicine. ${ }^{23,24,27}$

\section{Academic Background}

One study found that neither undergraduate gradepoint average nor scores on the MCAT science problems were predictive for a choice of family medicine. ${ }^{22}$ College major also did not relate to choice of a residency in family medicine compared with internal medicine. ${ }^{28}$ Higher undergraduate science grade-point averages increased the probability of choice of family practice, but higher scores on the MCAT chemistry test decreased the probability. ${ }^{23}$ The mixed results of these few studies do not permit any firm conclusions about academic background and its relationship to specialty choice.

\section{Personality}

There is slight evidence that those in family medicine are more likely to be "feeling" in the MyersBriggs classification, less authoritarian, and more humanistic. ${ }^{21,29,30}$ Based both on the few studies done and the minimal results, it would seem that we know little more about personality type and specialty choice than we did 8 years ago.

\section{Values and Knowledge on Entry to Medical School}

Three studies provide some evidence that values on entry to medical school that are congruent with a choice of family medicine are related to an eventual choice of the specialty. Expectations of high income in a medical career were negatively related to plans for family practice, as was an interest in re- 
search. ${ }^{23}$ Conversely, agreement that primary care physicians are more important than specialists increased the chances of plans for family practice. Students at a school with a relatively low rate of producing primary care graduates were significantly less likely to identify family medicine as a specialty with board certification than were students at a school with high rates of primary care graduates. ${ }^{31}$ Finally analysis of medical school application essays indicated that a mention of contact with a particular specialty was related to entering the same or a "similar" specialty. ${ }^{32}$

\section{Career Intentions on Entry to Medical School}

Specialty preference. All 3 studies that looked at preference for family medicine at entry to medical school found that it increased the likelihood of eventual plans for family practice. ${ }^{22,23,33}$

Five studies documented the changing levels of interest in family medicine over the course of medical school. All found that initial interest declined. ${ }^{17,24,34-36}$ One retrospective study of family physicians did find that a majority reported interest increasing during medical school. ${ }^{37}$ Another study demonstrated that the ability to attract students to family medicine during medical school is positively related to the family medicine output of the school.

The studies described above looked at initial interest after the student had been admitted to medical school, which is not necessarily consistent with a stated interest before admission. Schneider et $\mathrm{al}^{32}$ analyzed application essays for students who were subsequently admitted and found that the stated goal of entering a particular specialty was not at all predictive of their eventual match; 11 students stated an intention to enter family medicine but only 1 did.

In a study using medical schools as the unit of analysis, Senf et $\mathrm{al}^{27}$ found that percentage of students interested in family medicine at matriculation was the most powerful predictor of the estimated number of students in a family medicine practice 6 years later, although the proportion of students interested in family medicine did increase over the course of medical school at three fourths of US medical schools.

All these studies support the conclusion that interest at matriculation is positively related to an eventual choice of family medicine. However, these studies also show that preferences for a particular specialty change considerably during the years of medical school, with attrition to other specialties among those initially interested in family medicine and significant recruitment to family medicine among students who had not planned on family medicine on entry to medical school. An important addition to the data available in 1995 is that there may not be any relationship between stated career intentions before admission to medical school and those indicated after admission.

Type of practice. The only study that looked at practice plans at matriculation found plans to practice in a rural setting or plans to practice in a socioeconomically disadvantaged area were related to interest in family medicine. ${ }^{24}$ It is difficult to draw any firm conclusions based on just one study; this is one of the areas needing additional research.

\section{School Characteristics}

Legislative Requirements and Mandates

Legislative mandates to produce primary care graduates are relatively recent, and little research has been done on potential effects of these efforts. ${ }^{3}$ One study did examine the impact of California's agreement with the state schools to increase their primary care graduates and found that choice of family medicine did increase from 1993 to $1998 .^{38}$ However, this study did not control for national trends, and during those same years the proportion of US graduates selecting family medicine nationally increased from $12.6 \%$ to $17.3 \%$ in 1997 and declined to $16 \%$ in $1998 .{ }^{8}$ Given these national trends, it is unclear whether the legislative mandate had any unique impact.

\section{Type of School}

Two studies found that public ownership of the school increased the likelihood of plans for family medicine, ${ }^{22,39}$ Campos-Outcalt and Senf ${ }^{40}$ found an interaction between ownership of the school and the history of required time in family medicine. Public schools that had required time in 1980-81 had the highest proportion of students going into family medicine 15 years later in 1995-96, followed by (1) private schools that had required time in 1980-81, (2) public schools introducing required time, (3) public schools with no required time, (4) private schools introducing required time, and (5) private schools with no required time. On the other hand, 2 studies did not find that ownership of the school predicted plans for family medicine. ${ }^{23,41}$ Using quality scores to weight the importance of these 
inconsistent results, it would seem that one can still conclude that public ownership is a predictor of production of graduates in family medicine (Mean quality scores for studies finding a positive relationship were 45.7 versus 32.5 for those finding a negative relationship).

\section{Mission}

Two studies suggest that a school's mission is related only indirectly to graduates' selection of family medicine. ${ }^{23,27}$

\section{Departmental Structure}

One study found that presence of a department of family medicine increased the likelihood of students planning a career in family medicine ${ }^{23}$; however, 2 other studies did not. ${ }^{39,40}$ Although all 3 of these studies have high quality scores, the 2 that did not find a relationship had the highest, leading to a tentative conclusion that department status is not directly related to production of family medicine graduates.

\section{School Research Funding}

Research funding has not been found to relate to choice of family practice ${ }^{23,27}$ Based on this somewhat limited body of research, it seems that research funding is not directly related to production of primary care graduates and, as suggested by Bland et al, ${ }^{9}$ most probably influences the characteristics and values of the faculty attracted to the school who in turn influence the attitudes of the students who are taught by them.

\section{Admission Policies}

Two studies have found that more primary care faculty on admissions committees ${ }^{27}$ and an admission preference for generalists were not related to increased numbers of family medicine graduates. ${ }^{23}$ Although these findings are counterintuitive, these data suggest that admissions policies or committees that favor generalism do not have an impact on the production of family physicians.

\section{Medical School Experiences}

Special Programs for Primary Care

Three of the programs reviewed in 1995 have published additional reports in the years since (University of New Mexico, University of Washington, Jefferson Medical College). All 3 continue to report higher proportions of graduates in family medicine from the special pathway than from the conventional curriculum. ${ }^{42-44}$

In addition to schools with special programs, one school was founded with the explicit goal of graduating primary care physicians for the rural areas in the state of Georgia. ${ }^{45}$ This school produces a proportion of graduates in family medicine well above the national average.

Thus, as in 1995, there is evidence that special programs lead to an increase in graduates in family medicine; however, we do not know which of the specific components of these programs may be producing this result.

\section{Unofficial Climate for Primary Care}

Although a medical school may have an official mission and curricula to support the choice of primary care, within the school there is an unofficial culture that may be working at cross-purposes. Two studies have looked at the existence and impact of negative attitudes expressed unofficially by faculty, residents, and students during the process of medical education. Both found that a majority of students report hearing negative comments about their specialty, ${ }^{35,46}$ and a small percentage report changing specialties because of this. It is clear from these studies that the unofficial culture of medical schools has an impact on specialty choice.

\section{First- and Second-Year Curricula}

Two studies looked at curricula in the first and second years. Neither found that courses in the first or second year were related to the choice of family medicine. ${ }^{23,39}$ The evidence continues to indicate minimal or no effect of early curriculum interventions.

\section{Third-and Fourth-Year Curricula and Experiences}

There have been a number of studies examining third year, required time; all but one $\mathrm{e}^{22}$ supported the positive relationship between required time in family medicine and selection of family medicine as a specialty. ${ }^{23,27,39,42,47}$ One study found lowered rates of students selecting family medicine if their internal medicine clerkship was ambulatory. ${ }^{48}$

\section{Faculty Composition}

Two multivariate studies since 1993 have not supported the conclusion that faculty composition (ie, the proportion of clinical faculty who are family physicians) is related to specialty choice. ${ }^{23,27}$ As 
with a number of other variables, it would seem that the relationship between faculty composition and specialty selection is indirect.

\section{Faculty Role Models}

Faculty role models have been examined in 3 studies. ${ }^{35,37,49}$ All found that having role models is related to specialty choice; in particular, negative role models were cited as a reason for switching away from family medicine. ${ }^{37}$ Despite the intuitive belief that positive role models should be important in specialty selection, these studies generally suggest that it is the negative experiences that have the most impact.

\section{Outcomes of Medical Education \\ Debt}

The relationship of debt to choice of family medicine remains unclear. Of the 4 studies that examined debt, 2 did not find a relationship, ${ }^{23,24}$ one found a threshold above which students were more likely to enter family medicine (but as debt increased above that point, it became less likely that they would enter family medicine ${ }^{22}$ ), and a fourth found a threshold below which there was increased choice of family medicine. ${ }^{33}$ These 4 studies have uniformly high quality scores; the relationship between debt and specialty choice remains unclear.

\section{Knowledge of Content and Characteristics of Specialties}

One study tracked attitudes toward family medicine from the first year of medical school through internship and found that overall attitudes became more positive until the third year and declined each year after that. ${ }^{17}$ Two studies found that students rejecting family medicine were concerned about a lack of prestige, low income potential, and low intellectual content of the specialty. ${ }^{24,37}$ Generalist orientation was found to predict choice of a family medicine residency at the point of selection of a residency. ${ }^{50}$ These studies suggest that perceptions about the characteristics and content of specialties influence specialty decisions, although the available research is extremely limited in scope.

\section{Career Intentions at Graduation}

Career intentions consistent with choice of family medicine include low pay expectations, ${ }^{22,24}$ and a practice in a rural or underserved location. ${ }^{24}$ Interest in research or an academic career are negatively related. ${ }^{22,24}$

\section{Discussion \\ Entry to Medical School: Student Characteristics, Values and Plans}

Many of the student characteristics that seemed related to specialty choice in 1995 are now less clearly important. Research in the last 8 years indicates that gender, age, marital status, and ethnicity are only weakly related to the choice of specialty. Academic background was never clearly related, and the recent literature has not changed that conclusion. In contrast, research continues to support the existence of a positive relationship between a student's rural background and choice of family medicine and a negative relationship between parents' socioeconomic status (education/ income) and choice of family medicine.

Career intentions at entry to medical school are predictive of eventual specialty choice; intending a career in family medicine or a practice in a rural or an underserved area are related to eventual choice of family medicine. Although these initial intentions are strongly predictive, there is considerable attrition over the course of medical school. However, recent research indicates that there is also recruitment, and it is the latter that accounts for most of the students who select family medicine residencies at the end of medical school. Another significant new finding is that stated goals to enter family medicine before entry to medical school are not related to eventual specialty choice, although reported contact with a specialty is.

Finally, student values or expectations on entry to medical school that are consistent with the choice of family medicine seem to predict eventual choice; students who believe primary care is important, have low income expectations, and do not plan for a career in research are more likely to eventually choose family medicine.

\section{School Characteristics}

The only school characteristic that currently seems related to choice of family medicine is public ownership of the school. Recent research does not confirm a relationship between specialty choice and the school's generalist mission, admissions policy or committee composition, structure (departmental family medicine unit), amount of research funding, or legislative mandate to produce generalists. 


\section{Medical School Experience}

Prior reviews found no evidence that curricula in the first and second years of medical school were related to the choice of family medicine, and research in the intervening 8 years confirms this result. Recent research on the effect of higher numbers of family medicine faculty does not support a conclusion that having a higher proportion of family medicine faculty is directly related to choice of the specialty. However, there are a number of medical school experiences that are consistently related to specialty choice. Large-scale programs with a goal of increasing the number of students entering primary care do seem to influence the number of students who select family medicine residencies and who practice family medicine. Required family medicine time in the third or fourth year is positively related to higher numbers of students selecting family medicine, and in the last 8 years, a longitudinal study has indicated that adding required time is followed by an increase in the percentage of students selecting family medicine. The informal culture or "hidden curriculum" created by the opinions and comments of students, residents, and faculty also influences students' specialty choice, although the evidence thus far has focused on the negative effects and does not indicate whether the overall impact is to increase or decrease the number of students entering family medicine. Finally, faculty role models in medical school are important, but serve as both positive and negative influences.

\section{Outcomes of Medical Education}

Over the course of medical school, many students acquire considerable financial debt. There has never been a clear-cut relationship between debt and specialty choice, and the more recent research indicates that if there is a relationship, it is complex. One of the inevitable outcomes of 4 years of medical education is that students develop perceptions, correct or not, about the content and characteristics of each specialty. These beliefs about the content and characteristics of specialties are consistently related to choice of specialty; students who reject family medicine are concerned about prestige, low income, and the breadth of knowledge required. Students who end their medical school career planning on a career in a disadvantaged or rural area, like students who begin their career with these intentions, are more likely to enter family medicine. Expectations for low income at graduation are also positively related to plans to enter family medicine; conversely, plans for an academic or research career are negatively related.

Given that the above is what we know at this point, unanswered questions include: (1) Why have we seen changes in the last 8 years? and (2) Where do we need to go next?

First, what should be made of apparent changes? For example, the characteristics and beliefs that students bring to medical school are important but perhaps less important than was previously thought. This apparent change may have been caused by the increasing use of more sophisticated multivariate analyses that help to identify those variables that are only indirectly related to specialty choice through a third variable. Specifically, older age and married status, which previously were found to be related to a choice of family medicine, may actually be part of a group of variables related to a desire for a rural practice location, which in turn is the more direct predictor of choice of family medicine. It is also possible that the more current study is less well done than those from which prior conclusions were drawn; quality scores for the prior studies were not available so this comparison could not be made.

Some of the change may reflect our increasingly precise measurement and understanding of the process of specialty choice. Research has long indicated that early career intentions are a good predictor of eventual specialty choice, but recent research on the group of students who do select family medicine residencies has revealed that the majority of students did not intend a career in family medicine when they entered medical school. This finding improves our understanding of the decision making process and reinforces the importance of the influence of medical education.

Some of the change may be real. In the past, being female was positively related to a choice of family medicine. However, this relationship may have been attributable to barriers that women encountered when they considered entering traditionally male specialties. As more women enter the profession of medicine and are admitted into the specialties historically dominated by men, this prior association may begin to disappear.

Finally, what are the next steps in a research agenda? Most of the topics that are a part of this review would be more informative if there were 
more research done in the area, especially if the research included multivariate analyses that allowed investigators to distinguish between the factors that are related to specialty choice primarily through an intervening variable and those factors that are related directly.

Two areas in particular that would benefit from more detailed and targeted research are the factors that influence specialty choice before medical school and the curricular components and informal culture that influence students during the process of medical education. For example, it is clear that required time in family medicine increases the number of graduates in family medicine; however, the aspects of this required time that are the most influential are less clear. Research on role models in medical school is relatively limited, yet the research that exists suggests that the influence of faculty is significant both within the formal curricula and in the "hidden" curricula. Much more information is needed about the messages given to students on an informal basis and the influence of these messages on specialty choice.

\section{References}

1. US Department of Health and Human Resources. Summary report of the graduate medical education national advisory committee, September 30, 1980. DHHS Publication no. (HRA) 81-651. Washington (DC): US Department of Health and Human Services; 1980.

2. Urban and rural population: 1900 to 1990 . Washington (DC): US Census Bureau, Department of Commerce; 1995. Available at: URL: http://www. census.gov/population/censusdata/urpop0090.txt.

3. Thomas CS, Henderson TM, Lipson LR, DiBiaso PA. A review of state legislation related to increasing the training, supply, recruitment and retention of generalist physicians: 1985-1991. DHHS Publication no. HRSA-HC-PC-94-2. Washington (DC): US Department of Health and Human Services, Bureau of Health Professions; 1994.

4. Anonymous. AAMC policy on the generalist physician. Acad Med 1993;68:1-6.

5. Bazell C, Kahn R. From the primary care organizations consortium's proposal to the Interdisciplinary Generalist Curriculum Project. Acad Med 2001;76(4 Suppl):S13-8.

6. Colwill JM, Perkoff GT, Blake RL Jr, Paden C, Beachler M. Modifying the culture of medical education: the first tree years of the RWJ Generalist Physician Initiative. Acad Med 1997;72:745-53.

7. Bland CJ, Starnaman S, Harris D, Henry R, Hembroff L. "No fear" curricular change: monitoring curricular change in the W. K. Kellogg Foundation's national initiative on Community Partnerships and Health Professions Education. Acad Med 2000;75: 623-33.

8. Data taken from the annual report of the results of the National Residency Matching Program published in the June issue of Academic Medicine 1993 to 2001 .

9. Bland CJ, Meurer LN, Maldonado G. Determinants of primary care specialty choice: a non-statistical meta-analysis of the literature. Acad Med 1995;7: $620-41$.

10. Meurer LN. Influence of medical school curriculum on primary care specialty choice: analysis and synthesis of the literature. Acad Med 1995;70:388-97.

11. Campos-Outcalt D, Senf J, Watkins A, Bastacky S. The effects of medical school curricula, faculty role models and biomedical research support on generalist physician careers: a review and quality assessment of the literature. Acad Med 1995;70:611-9.

12. Reed VA, Jernstedt CR, Reber ES. Understanding and improving medical student specialty choice: a synthesis of the literature using decision theory as a referent. Teach Learn Med 2001;13:117-29.

13. Rosenthal MP, Rabinowitz HK, Diamond JJ, Markham FW Jr. Medical students' specialty choice and the need for primary care. Our future. Prim Care 1996;23:155-67.

14. Rosenthal MP. Half full or half empty? A look at family practice specialty choice after strolling through the 1994 match. Fam Med 1994;26:524-7.

15. Bickel J, Ruffin A. Gender-associated differences in matriculating and graduating medical students. Acad Med 1995;70:552-9.

16. Cooksey JA, Dry LR, Harman CP, Killian CD. Primary care residency selection and completion-the Illinois experience, 1988-1995. Acad Med 1997;72: S106-8.

17. Mann MP. Attitudes toward and subsequent career choice of family practice: a weak relationship. Fam Med 1994;26:504-8.

18. Litzelman DK, Marriott DJ. Impact of increasing women medical school matriculates on primary care residency training choices: a five-year perspective. Acad Med 1996;71:S13-5.

19. Hojat M, Robeson MR, Veloski JJ, Blacklow RS, Xu G, Gonnella JS. Gender comparisons prior to, during and after medical school using two decades of longitudinal data at Jefferson Medical College. Eval Health Prof 1994;17:290-306.

20. Xu G, Rattner SL, Veloski JJ, Hojat M, Fields SK, Barzansky B. A national study of the factors influencing men and women physicians' choices of primary care specialties. Acad Med 1995;70:398-404.

21. Stilwell NA, Wallick MM, Thal SE, Burleson JA. Myers-Briggs type and medical specialty choice: a new look at an old question. Teach Learn Med 2000;12:14-20. 
22. Colquitt WL, Zeh MC, Killian CD, Cultice JM. Effect of debt on U. S. medical school graduates' preferences for family medicine, general internal medicine, and general pediatrics. Acad Med 1996;71: $399-411$.

23. Kassebaum DG, Szenas PL, Schuchert MK. Determinants of the generalist career intentions of 1995 graduating medical students. Acad Med 1996;71: 197-209.

24. Bowman M, Haynes RA, Rivo ML, Killian CD, Davis H. Characteristics of medical students by level of interest in primary care. Fam Med 1996;28:713-9.

25. Xu G, Veloski JJ, Barzansky B. Comparisons between older and usual-aged medical school graduates on the factors influencing their choices of primary care specialties. Acad Med 1997;72:1003-7.

26. Pamies RJ, Lawrence LE, Helm EG, Strayhorn G. The effects of certain student and institutional characteristics on minority medical student specialty choice. J Natl Med Assoc 1994;86:136-40.

27. Senf JH, Campos-Outcalt D, Watkins AJ, Bastacky S, Killian C. A systematic analysis of how medical school characteristics relate to graduates' choices of primary care specialties. Acad Med 1997;72:524-33.

28. Elam C. Primary care specialty selection: application of a vocational behavior theory. Fam Med 1995;27: 249-54.

29. Merrill JM, Laux LF, Lorimor R, Thornby JI, Vallbona $\mathrm{C}$. Using attribution theory models to predict senior medical students' perceptions of patients and career choice. Acad Med 1996;71(1 Suppl):S67-9.

30. Coutts-van Dijk LC, Bray JH, Moore S, Rogers J. Prospective study of how students' humanism and psychosocial beliefs relate to specialty matching. Acad Med 1997;72:1106-8.

31. Grayson MS, Newton DA, Whitley TW. First-year medical students' knowledge of and attitudes toward primary care careers. Fam Med 1996;28:337-42.

32. Schneider FD, Hosokawa MC, Campbell JD. Medical school application essays and specialty choice. Fam Med 1994;26:509-12.

33. Rosenthal MP, Marquette PA, Diamond JJ. Trends along the debt-income axis: implications for medical students' selections of family practice careers. Acad Med 1996;71:675-7.

34. Haviland MG, Werner LS, Behrens L, Killian CD. Preparing for health care reform and an LCME site visit: addressing the generalist-non-generalist imbalance. Acad Med 1995;70:298-304.

35. Hearst N, Shore WB, Hudes ES, French L. Family practice bashing as perceived by students at a university medical center. Fam Med 1995;27:366-70.

36. Schafer S, Shore W, French L, Tovar J, Hughes S, Hearst N. Rejecting family practice: why medical students switch to other specialties. Fam Med 2000; $32: 320-5$.
37. Xu G, Hojat M, Brigham TP, Veloski JJ. Factors associated with changing levels of interest in primary care during medical school. Acad Med 1999;74: 1011-5.

38. Podnos YD, Campbell B, Wilson SE, Williams RA. Patterns of graduating medical student career selections from 1993 to 1998 and their effect on surgery as a career choice. Arch Surg 1999;134:876-81.

39. Mengel MB, Davis AB. Required first-year generalist clinical experience courses and their relationship to career choice: the critical effect of family medicine involvement. Fam Med 1995;27:652-7.

40. Campos-Outcalt D, Senf J. A longitudinal, national study of the effect of implementing a required thirdyear family practice clerkship or a department of family medicine on the selection of family medicine by medical students. Acad Med 1999;74:1016-20.

41. Cummings M, Ennis M. A model for improving generalist physician output: the osteopathic experience. Acad Med 1995;70:S57-63.

42. Mennin SP, Kalishman S, Friedman M, Pathak D, Snyder J. A survey of graduates in practice from the University of New Mexico's conventional and community-oriented, problem-based tracks. Acad Med 1996;71:1079-89.

43. Phillips TJ, Rosenblatt RA, Schaad DC, Cullen TJ. The long-term effect of an innovative family physician curricular pathway on the specialty and location of graduates of the University of Washington. Acad Med 1999;74:285-8.

44. Rabinowitz HK, Diamond JJ, Markham FW, Hazelwood CE. A program to increase the number of family physicians in rural and underserved areas: impact after 22 years. JAMA 1999;281:255-60.

45. Ackermann RJ, Comeau RW. Mercer University School of Medicine: a successful approach to primary care medical education. Fam Med 1996;28: 395-402.

46. Hunt DD, Scott C, Zhong S, Goldstein E. Frequency and effect of negative comments ("badmouthing") on medical students' career choices. Acad Med 1996;71:665-9.

47. Senf JH, Campos-Outcalt D. The effect of a required third-year family medicine clerkship on students' attitudes: value indoctrination and value clarification. Acad Med 1995;70:142-8.

48. Bauer RL, Venkatachalam HM, Forrester RH, Harris GD, Diehl AK. The effect of an ambulatory internal medicine rotation on students' career choices. Acad Med 1997;72:147-9.

49. Wright S, Wong A, Newill C. The impact of role models on medical students. J Gen Intern Med 1997; 12:53-6.

50. Nasmith L, Rubenstein H, Goldstein H, Sproule D, Franco ED, Tellier P. Predicting who will choose a family medicine residency. Acad Med 1997;72: $908-12$. 